\title{
ULM'S THEOREM FOR PARTIALLY ORDERED STRUCTURES RELATED TO SIMPLY PRESENTED ABELIAN $p$-GROUPS \\ BY
}

\author{
LAUREL A. ROGERS
}

\begin{abstract}
If we have an abelian $p$-group $G$, a multiplication by $p$ for each element of $G$ is defined by setting $p x=x+x+\cdots+x$, where $p$ is the number of terms in the sum. If we forget about the addition on $G$, and just keep the multiplication by $p$, we have the algebraic structure called a $p$-basic tree. A natural partial order can be defined, the graph of which is a tree with 0 as root. A p-basic tree generates a simply presented abelian $p$-group, and provides a natural direct sum decomposition for it. Ulm invariants may be defined directly for a $p$-basic tree so that they are equal to the Ulm invariants of the corresponding group. A central notion is that of a stripping function between two $p$-basic trees. Given a stripping function from $X$ onto $Y$ we can construct an isomorphism between the groups corresponding to $X$ and $Y$; in particular, $X$ and $Y$ have the same Ulm invariants. Conversely, if $X$ and $Y$ have the same Ulm invariants, then there is a map from $X$ onto $Y$ that is the composition of two stripping functions and two inverses of stripping functions. These results constitute Ulm's theorem for $p$-basic trees, and provide a new proof of Ulm's theorem for simply presented groups.
\end{abstract}

1. Introduction. In 1933, Ulm [7] characterized countable reduced abelian $p$-groups in terms of functions from ordinals to cardinals defined by $f(\alpha, G)$ $=\operatorname{dim} p^{\alpha} G[p] / p^{\alpha+1} G[p]$, the Ulm invariants of $G$. This result was extended by Kolettis [6] in 1960 to direct sums of such groups, and by Hill [4], [5] in 1967 to the class of all totally projective groups. In 1969, Crawley and Hales [1] proved Ulm's theorem for reduced simply presented $p$-groups ( $T$-groups, in their terminology), and showed that the class of reduced simply presented $p$-groups coincides with the class of all totally projective groups.

The principal object of study in this paper is the $p$-basic tree, which is a set upon which multiplication by a prime $p$ is defined. A $p$-basic tree generates a simply presented $p$-group, and provides a natural direct sum decomposition for it. Conversely, any such group contains a $p$-basic tree which generates it. Ulm invariants can be defined directly for a $p$-basic tree so that they are equal to the Ulm invariants of the corresponding group.

A central notion introduced here is that of a stripping function between two $p$-basic trees. Given a stripping function, we can construct an isomorphism between the corresponding groups (Theorem 2). If a map between two

Received by the editors January 5, 1976.

AMS (MOS) subject classifications (1970). Primary 20K10; Secondary 06A10. 
$p$-basic trees exists which is the composition of a stripping function and the inverse of a stripping function (call such a map a strip-graft), then the natural direct sum decompositions of the corresponding groups have isomorphic refinements (Theorem 3). A principal result (Theorem 4) is that two p-basic trees generate isomorphic groups if and only if a map between them exists which is the composition of two strip-grafts. We finish with a version of Ulm's theorem for $p$-basic trees (Theorem 5), which gives a new proof of Ulm's theorem for simply presented groups.

2. Preliminaries. Throughout the discussion, the letter $p$ shall denote a fixed but arbitrary prime, and "group" shall mean "abelian $p$-group". We shall conform to standard terminology and notation as used, for example, in [3].

If we have a group $G$, a multiplication by $p$ for each element of $G$ is defined by setting $p x=x+x+\cdots+x$, where $p$ is the number of terms in the sum. This is a most important structure on $G$; in particular, it has the property that given $g$ in $G$, there is an integer $n$ with $p^{n} g=0$. If we forget about the addition on $G$, and just keep the multiplication by $p$, we have the algebraic structure which we shall call a $p$-basic tree.

Definition 1. A $p$-basic tree is a set $X$ together with a multiplication by $p$ for elements of $X$ such that:

(1) $p x \in X$ for each $x$ in $X$,

(2) there is an element 0 in $X$ for which $p 0=0$, and

(3) for each nonzero element $x$ in $X$, there is a positive integer $n$ such that $p^{n} x=0$.

If $x \in X$, the least integer $n$ such that $p^{n} x=0$ is the exponent of $x$. The relation $>$ on $X$ is defined as follows: for elements $x, y$ of $X$, if there is a positive integer $m$ such that $p^{m} x=y$, then $x>y$. Clearly, the relation $\geqslant$ is a partial order. The graph of $X$ under this partial order is a tree with 0 as root. An atom of $X$ is a nonzero element $z$ with the property that, if $z \geqslant x$, then $x=z$ or $x=0$. For each $x$ in $X$, set $C(x)=\{y \in X: y \geqslant x$ or $y<x\}$. A subset $S$ of $X$ is a subtree of $X$ if, for each $s$ in $S$, the element ps is in $S$. A collection $\left\{S_{i}\right\}$ of subtrees of $X$ is disjoint if $S_{i} \neq S_{j}$ implies $S_{i} \cap S_{j}=\{0\}$. If $\left\{S_{i}\right\}$ is a disjoint collection of subtrees of $X$ such that $X=\cup S_{i}$, then $X$ is the direct sum of its subtrees $S_{i}$ and we write $X=\bigoplus S_{i}$. Note that if $x \in X$, then $C(x)$ is a subtree, and if $A$ is the set of atoms of $X$, then $X=\bigoplus_{a \in A} C(a)$. The group whose generators are elements of $X$, subject to the relations $p x=y$, is the group generated by $X$, and shall be denoted by [X]. The next definition is taken from [3].

Definition 2. A group $G$ is simply presented if it has a set $X$ of generators and a set of relations such that:

(1) for every $x$ in $X, x \neq 0$ in $G$,

(2) if $x, y$ are distinct elements in $X$, then $x \neq y$ in $G$, and

(3) all relations are of the form $p x=0$ or $p x=y$ where $x, y \in X$. 
The set $X$ is a $p$-basis of $G$. A $p$-basis can also be characterized in the following way [2], [8]: a subset $X$ of $G$ such that if $x \in X$ and $p x \neq 0$, then $p x \in X$, and every element in $G$ can be written uniquely in the form $\Sigma_{x \in X} n_{x} x$ with $0 \leqslant n_{x}<p$. It is shown in [2] that a group is simply presented iff it has a $p$-basis. The representation of an element in the form $\Sigma n_{x} x$ will be referred to as the unique representation of the element as a linear combination of elements of $X$. It is clear that if $X$ is a $p$-basic tree, then $[X]$ is a simply presented group with $p$-basis $X \sim\{0\}$; conversely, if a group is simply presented with a $p$-basis $X$, then $X \cup\{0\}$ is a $p$-basic tree.

Observe now that if $X$ is a $p$-basic tree and $\left\{S_{i}\right\}$ a collection of subtrees such that $X=\bigoplus S_{i}$, then $[X]=\bigoplus\left[S_{i}\right]$. Consequently, if $A$ is the set of atoms of $X$, the decomposition $[X]=\bigoplus_{a \in A}[C(a)]$ shall be referred to as the natural direct sum decomposition of $[X]$ given by $X$.

Definition 3. Let $X$ be a $p$-basic tree. Set $p^{0} X=X$, and for any ordinal $\beta$, set $p^{\beta} X=\bigcap_{\alpha<\beta} p\left(p^{\alpha} X\right)$. An element $x$ of $X$ has height $\alpha$ if $x \in p^{\alpha} X \sim$ $p^{\alpha+1} X$; we shall denote the height of $x$ by $h x$. If $x \in p^{\alpha} X$ for every $\alpha$, then we write $h x=\infty$, where $\infty$ is considered to be larger than any ordinal. There must be some ordinal $\lambda$ for which $p^{\lambda} X=p^{\lambda+1} X$; the least such $\lambda$ is the length of $X$. If $p^{\lambda} X=0$, then $X$ is reduced. Henceforth we shall assume that all $p$-basic trees are reduced. A useful property [1] of simply presented groups is that if $b=\sum_{i=1}^{k} r_{i} x_{i}$ is the unique representation of a nonzero element $b$ as a linear combination of elements of $X$, then $b \in p^{\alpha} G$ iff $x_{i} \in p^{\alpha} G$ for $i=$ $1, \ldots, k$. This can be strengthened.

Proposition 1. Suppose $X$ is a p-basic tree, and $b$ is a nonzero element of $[X]$ whose representation as a linear combination of elements of $X$ is $b=$ $\sum_{i=1}^{k} r_{i} x_{i}$. If $h x_{i}$ represents the height in $X$ of $x_{i}$, then the height of $b$ in the group $[X]$ is $\min \left\{h x_{1}, \ldots, h x_{k}\right\}$.

PRoof. It suffices to verify that $p^{\alpha} X=X \cap p^{\alpha}[X]$ for any ordinal $\alpha$; this is accomplished by an induction on $\alpha$.

Definition 4. Two $p$-basic trees $X$ and $Y$ are isomorphic if there is a height-preserving bijection $\eta: X \rightarrow Y$ such that $\eta(p x)=p \eta(x)$ for every $x$ in $X$; the map $\eta$ is an isomorphism. If the groups $[X]$ and $[Y]$ are isomorphic, then $X$ and $Y$ are equivalent. Obviously, isomorphic $p$-basic trees are equivalent.

3. Ulm invariants and stripping functions. Recall that, for a group $G$ and an ordinal $\alpha$, the $\alpha$ th Ulm invariant of $G$ is the cardinal number

$$
f(\alpha, G)=\operatorname{dim} p^{\alpha} G[p] / p^{\alpha+1} G[p] .
$$

The corresponding notion for $p$-basic trees is the following.

Definition 5. Let $X$ be a $p$-basic tree of length $\lambda$, and $\alpha$ an ordinal such that $\alpha<\lambda$. Define $U(\alpha, X)=\{x \in X: h x=\alpha$ and $h(p x)>\alpha+1\}$. De- 
note by $A$ the set of elements of $X$ with height $\alpha+1$, and suppose $z \in A$. Choose an element in $p^{-1} z$ with height $\alpha$ and denote it by $z^{\prime}$. Set $D(z)=\{y$ $\in p^{-1} z$ : $h y=\alpha$ and $\left.y \neq z^{\prime}\right\}$. We now define the $\alpha$ th Ulm invariant of $X$ to be the cardinal number

$$
f(\alpha, X)=|U(\alpha, X)|+\left|\bigcup_{z \in A} D(z)\right| \text {. }
$$

Proposition 2. Let $X$ be a p-basic tree of length $\lambda$, and $\alpha$ an ordinal such that $\alpha<\lambda$. Then $f(\alpha, X)=f(\alpha,[X])$.

Proof. Denote the group $p^{\alpha}[X][p]$ by $G$, and its subgroup $p^{\alpha+1}[X][p]$ by $H$. For each $x$ in $U(\alpha, X)$, there is an element $u$ in $p^{\alpha} X$ with $p^{2} u=p x$, so $x-p u+H$ is a nonzero element of $G / H$. Let $B_{1}=\{x-p u+H: x \in$ $U(\alpha, X), u \in p^{\alpha} X$, and $\left.p^{2} u=p x\right\}$. For $z$ in $A$ (where $A$ is the set of elements of height $\alpha+1)$, and $y$ in $D(z)$, the element $y-z^{\prime}+H$ is a nonzero element of $G / H$. Let $B_{2}=\left\{y-z^{\prime}+H: z \in A, y \in D(z)\right\}$. We proceed to show that $B_{1} \cup B_{2}$ is a basis of $G / H$, considered as a vector space over $Z(p)$.

To see that $B_{1} \cup B_{2}$ is independent, suppose

$$
\begin{aligned}
\sum_{i=1}^{n} r_{i}\left(x_{i}-p u_{i}+H\right)+ & \sum_{i=1}^{k} t_{i 1}\left(y_{i 1}-z_{1}^{\prime}+H\right) \\
& +\cdots+\sum_{i=1}^{k^{\prime}} t_{i m}\left(y_{i m}-z_{m}^{\prime}+H\right)=0
\end{aligned}
$$

in $G / H$, where each $r_{i}$ and each $t_{i j}$ is a nonnegative integer less than $p$. This implies that in $[X]$, the element

$$
\sum_{i=1}^{n} r_{i} x_{i}+\sum_{i=1}^{k} t_{i 1} y_{i 1}+\cdots+\sum_{i=1}^{k^{\prime}} t_{i m} y_{i m}-\left(\sum_{i=1}^{k} t_{i 1}\right) z_{1}^{\prime}-\cdots-\left(\sum_{i=1}^{k^{\prime}} t_{i m}\right) z_{m}^{\prime}
$$

has height at least $\alpha+1$. Since all elements from $X$ in this expression are distinct, each $r_{i}=t_{i j}=0$.

To see that $B_{1} \cup B_{2}$ spans $G / H$, suppose $v+H$ is a nonzero element of $G / H$, and $v=\sum_{i=1}^{n} r_{i} x_{i}$ is its representation in $[X]$ as a linear combination of elements of $X$. Assume that any element in $G$ whose representation contains less than $n$ terms is in the linear span $S$ of $B_{1} \cup B_{2}$ in $G / H$. In the expression for $v$, if any $p x_{i}=0$, then $v-r_{i} x_{i}$ satisfies the induction hypothesis, and $x_{i}+H$ is equal to an element of $B_{1}$, so $v+H \in S$. If no $p x_{i}=0$, then in the set $\left\{x_{1}, \ldots, x_{n}\right\}$ there must be at least two elements $x_{i}$ and $x_{j}$ for which $p x_{i}=p x_{j}$; say $p x_{1}=\cdots=p x_{k}=z$. If $h z>\alpha+1$, there is $u$ in $p^{\alpha} X$ such that $p^{2} u=z$, and so

$$
v=r_{1}\left(x_{1}-p u\right)+\cdots+r_{k}\left(x_{k}-p u\right)+\sum_{i=1}^{k} r_{i}(p u)+\sum_{i=k+1}^{n} r_{i} x_{i} .
$$

Then $v-\sum_{i=1}^{k} r_{i}\left(x_{i}-p u\right)$ satisfies the induction hypothesis, and each $x_{i}-p u$ 
$+H$ is equal to an element of $B_{1}$, so $v+H \in S$. If $h z=\alpha+1$, write

$$
v=r_{1}\left(x_{1}-z^{\prime}\right)+\cdots+r_{k}\left(x_{k}-z^{\prime}\right)+\left(\sum_{i=1}^{k} r_{i}\right) z^{\prime}+\sum_{i=k+1}^{n} r_{i} x_{i} \text {. }
$$

Since $x_{i}-z^{\prime}+H \in B_{2}$, a similar argument shows that $v+H \in S$.

Definition 6. A $p$-basic tree is fully stripped if, whenever there are distinct elements $x$ and $y$ with $p x=p y$, then $h(p x)$ is a limit ordinal or $\infty$.

Definition 7. Let $X$ and $Y$ be $p$-basic trees and $\sigma: X \rightarrow Y$ a bijection. Then $\sigma$ is a stripping function if

(1) $\sigma$ preserves heights, and

(2) if $\sigma(p x) \neq p \sigma(x)$, then $p \sigma(x)=0$. A bijection $\xi$ is a strip-graft if there is a $p$-basic tree $Z$, a stripping function $\sigma: X \rightarrow Z$, and a stripping function $\psi$ : $Y \rightarrow Z$ such that $\xi=\psi^{-1} \sigma$. An easy consequence of the definition is the following.

Proposition 3. Suppose $X$ and $Y$ are p-basic trees and $\sigma: X \rightarrow Y$ is $a$ stripping function. Let $a$ be an atom of $X$, and set $N(a)=\{x \in C(a)$ : $p \sigma(x) \neq \sigma(p x)\}$. Then $\sigma a$ is an atom of $Y$, and $\sigma x$ is an atom of $Y$ for each $x$ in $N(a)$. Furthermore, the image of the subtree $C(a)$ is

$$
\sigma[C(a)]=\bigoplus_{x \in N(a)} C(\sigma x) \oplus C(\sigma a) .
$$

DEFINITION 8. Let $X$ be a $p$-basic tree and $z$ a nonzero element of $X$. A subset $D$ of $p^{-1} z$ is dense in $p^{-1} z$ if, given an ordinal $\alpha<h z$, there is an element $y$ in $D$ with $\alpha<h y$.

LemMa 1. Let $X$ be a $p$-basic tree, and $\sigma$ a bijection of $X$ onto a set $Y$. Suppose multiplication by $p$ is defined on $Y$ so that either $p \sigma(x)=\sigma(p x)$, or $p \sigma(x)=\sigma(0)$. Then $Y$ is a $p$-basic tree, and $\sigma$ is a stripping function iff for every nonzero element $z$ in $X$, the set $\left\{y \in p^{-1} z: p \sigma(y)=\sigma(p y)\right\}$ is dense in $p^{-1} z$.

Proposition 4. Let $X$ be a p-basic tree. Then there is a fully stripped p-basic tree $X^{\prime}$ and a stripping function $\sigma: X \rightarrow X^{\prime}$.

Proof. Define *, a new multiplication by $p$ for $X$, as follows: $p * x=p x$, if $h(p x)$ is a limit ordinal or if there is some $z$ in $X$ for which $x=z^{\prime}$, and $p * x=0$ otherwise. With this multiplication, $X$ is a fully stripped $p$-basic tree, which shall be denoted $X^{\prime}$. Lemma 1 shows that the identity function $\sigma$ is a stripping function.

THEOREM 1. If $X$ and $Y$ are $p$-basic trees and $\sigma: X \rightarrow Y$ is a stripping function, then $X$ and $Y$ have the same Ulm invariants.

Proof. Let $\alpha$ denote an arbitrary ordinal, and $A$ the set of elements of $X$ with height $\alpha+1$. The set $A^{\prime}$ of elements of $Y$ with height $\alpha+1$ coincides with $\sigma[A]$. The result follows from the fact that there is a one-to-one 
correspondence between the sets $U(\alpha, X) \cup \cup_{z \in A} D(z)$ and $U(\alpha, Y) \cup$ $\bigcup_{z \in A} D(\sigma z)$.

In view of Theorem 1, it is a consequence of Ulm's theorem for simply presented groups that $[X]$ and $[Y]$ are isomorphic. However, we can prove this result directly in a more general setting, and use it to prove Ulm's theorem.

Theorem 2. Let $X$ and $Y$ be p-basic trees and suppose $\sigma: X \rightarrow Y$ is a stripping function. Then $X$ and $Y$ are equivalent.

Proof. Define the function $d$ on $X$ as follows: set $d(0)=0$, and if the exponent of $x$ is $k+1$, set $d(x)=d(p x)$ if $p \sigma(x)=\sigma(p x)$, and set $d(x)=$ $d(p x)+1$ if $p \sigma(x) \neq \sigma(p x)$. We use $d$ to define inductively a function $\pi$ : $X \rightarrow X$ such that for each $x$ in $X$ we have $p \pi(x)=\pi(p x)$ iff $p \sigma x=\sigma(p x)$, and such that: (1) $h(\pi x) \geqslant h x,(2)$ there is a nonnegative integer $n$ for which $\pi^{n}(x)=0$, (3) either $p \pi x=\pi p x$ or $p \pi x=p x$, and (4) if $\pi x \neq 0$, then $d(\pi x)$ $<d(x)$. The construction of $\pi$ is as follows. If $d(x)=0$ or if $p x=0$, set $\pi x=0$. Assume $\pi$ has been defined to satisfy (1)-(4) for all $y$ such that $d(y)<k$, and for all $y$ such that $d(y)=k$ and $p^{m-1} y=0$, where $k$ and $m$ are positive integers. Suppose $x$ is an element of $X$ whose exponent is $m$, and for which $d(x)=k$. If $p \sigma x \neq \sigma p x$, then by Lemma 1 there is an element $y$ of $X$ with $p y=p x, h y \geqslant h x$, and $\sigma p y=p \sigma y$. Since $d(y)=d(p y)=d(p x)<$ $d(x), \pi y$ has been defined. Set $\pi x=y$. If $p \sigma x=\sigma p x$, then $d(x)=d(p x)$, but the exponent of $p x$ is $m-1$; hence $\pi(p x)$ has been defined. If $\pi(p x)=0$ set $\pi x=0$. Otherwise, note $h(\pi(p x)) \geqslant h(p x)>h x$, so there is an element $u$ of $X$ with $p u=\pi(p x)$ and $h u \geqslant h x$. If $p \sigma u=\sigma p u$, then $\pi u$ has been defined; set $\pi x=u$. On the other hand, if $p \sigma u \neq \sigma p u$, then by Lemma 1 there is an element $w$ of $X$ with $p w=p u, h w \geqslant h u$, and $\sigma(p w)=p \sigma w$. Set $\pi x=w$.

We use $\pi$ to define yet another function $\pi^{\prime}: X \rightarrow[X]$ by setting $\pi^{\prime}(x)=x$ $-\pi x$. Denote the image of $X$ in $[X]$ by $X^{\prime}$. Then $X^{\prime}$ is a $p$-basic tree, and $\pi^{\prime}$ : $X \rightarrow X^{\prime}$ is a stripping function. Furthermore, $X^{\prime} \sim\{0\}$ is a $p$-basis of $[X]$ (to see this, note if $g=\sum_{i=1}^{k} r_{i} x_{i}$ in $[X]$, with each $x_{i}$ in $X$, a representation of $g$ as a linear combination of elements of $X^{\prime}$ is

$$
g=\sum_{i=1}^{k}\left[r_{i}\left(x_{i}-\pi x_{i}\right)+\cdots+r_{i}\left(\pi^{m-1} x_{i}-\pi^{m} x_{i}\right)\right],
$$

where $m$ is large enough so that $\pi^{m} x_{i}=0$ for all $\left.i=1, \ldots, k\right)$.

Finally, observe that $Y$ and $X^{\prime}$ are isomorphic under the map $\eta: Y \rightarrow X^{\prime}$ defined by $\eta(\sigma x)=\pi^{\prime}(x)$.

THEOREM 3. Let $X$ and $Y$ be $p$-basic trees and $\xi: X \rightarrow Y$ a strip-graft. Then the natural direct sum decompositions of $[X]$ and $[Y]$ have isomorphic refinements. 
Proof. Let $Z$ be the $p$-basic tree with $\sigma: X \rightarrow Z$ and $\psi: Y \rightarrow Z$ the stripping functions such that $\xi=\psi^{-1} \sigma$. Then the set $T$ of atoms of $Z$ is $T=\{\sigma a: a \in A\} \cup\{\sigma x: p \sigma x \neq \sigma p x\}$, where $A$ is the set of atoms of $X$. Consequently, $[Z]=\bigoplus_{t \in T}[C(t)]$ is isomorphic to a refinement of $[X]=$ $\bigoplus_{a \in A}[C(a)]$. Similarly, $[Z]$ is isomorphic to a refinement of $[Y]$.

4. $p$-basic trees with the same Ulm invariants. In what follows, we shall be interested in $p$-basic trees with the same Ulm invariants, and because of Theorem 1 and Proposition 4, we may restrict our attention to fully stripped $p$-basic trees.

Definition 9. Suppose $X$ and $Y$ are $p$-basic trees and $\theta: X \rightarrow Y$ is a height-preserving bijection which has the property that $\theta(p x)=p \theta x$, except possibly when both $h(p x)$ and $h(p \theta x)$ are limit ordinals or $\infty$. Then we shall say that $\theta$ is a $T$-function.

Observe that the composition of two $T$-functions is a $T$-function; the inverse of a $T$-function is a $T$-function; and if both $X$ and $Y$ are fully stripped, and $\sigma: X \rightarrow Y$ is a stripping function, then both $\sigma$ and $\sigma^{-1}$ are $T$-functions.

For the purposes of the next several definitions and propositions, we shall assume that $X$ and $Y$ are fully stripped p-basic trees, and the symbol $\theta$ shall be reserved for a $T$-function.

Proposition 5. The $p$-basic trees $X$ and $Y$ have the same Ulm invariants iff there is a $T$-function $\theta: X \rightarrow Y$.

Proof. Suppose that $X$ and $Y$ have the same Ulm invariants; this is equivalent to the possession of bijections $\eta_{\alpha}: U(\alpha, X) \rightarrow U(\alpha, Y)$ for each ordinal $\alpha$. Note if $x$ is a nonzero element of $X$ with height $\alpha$ and $x \notin$ $U(\alpha, X)$, then there is a least positive integer $n$ such that $p^{n} x \in U(\zeta, X)$ for the ordinal $\zeta=\alpha+n$. Thus in $Y$, there is an element $y$ such that $p^{n} y=$ $\eta\left(p^{n} x\right)$; since $Y$ is fully stripped, $y$ is unique. The $T$-function $\theta$ is defined as follows: set $\theta(0)=0$, set $\theta(x)=\eta_{\alpha}(x)$ if $x \in U(\alpha, X)$, and set $\theta(x)=Y$ if $x \notin U(\alpha, X)$ and $y$ is as described above. The converse is easily verified. $\square$

Our immediate goal is to describe a $T$-function in terms of stripping functions. A certain partition of $X$ will be necessary.

Definition 10. Let $E=\{x \in X: p \theta x=\theta p x\}$. Suppose $S$ is a subset of $X$ which is the disjoint union of subsets $K, L, S \cap E$, and $A$, and $e$ is a function from $S$ to the nonnegative integers such that $e(0)=0$, and for nonzero $x$ in $S$,

(1) if $x \in K$ then $p x \in S$ and $e(x)=e(p x)+1$,

(2) if $x \in L$ then $\theta^{-1}(p \theta x) \in S$ and $e(x)=e\left(\theta^{-1}(p \theta x)\right)+1$,

(3) if $x \in S \cap E$ then $p x \in S$ and $e(x)=e(p x)+1$, and

(4) if $x \in A$ then $e(x)=1$. 
Under these conditions, we shall say that $(S, e, K, L, A)$ is a suitably partitioned subset of $X$ (or simply that $S$ is suitably partitioned). If $(S, e, K, L, A)$ and $\left(S^{\prime}, e^{\prime}, K^{\prime}, L^{\prime}, A^{\prime}\right)$ are two suitably partitioned subsets of $X$, we shall say $S \preccurlyeq S^{\prime}$ if $S \subset S^{\prime}, K \subset K^{\prime}, L \subset L^{\prime}, A \subset A^{\prime}$, and $e^{\prime}$ is an extension of $e$. Clearly, $\preccurlyeq$ is a partial order.

Definition 11. If $(S, e, K, L, A)$ is a suitably partitioned subset of $X$, we may construct a larger suitably partitioned subset by adjoining elements $x$ in $E$ for which $p x \in S$, and then defining $e(x)=e(p x)+1$ for such elements. Denote by $S^{*}$ the largest superset of $S$ which can be obtained in this way, and let $e^{*}$ denote the corresponding extension of $e$. Then $\left(S^{*}, e^{*}, K, L, A\right)$ is a suitably partitioned subset of $X$.

Lemma 2. Let $(S, e, K, L, A)$ be a suitably partitioned subset of $X$, and $F$ a finite subset of $X$ which is the disjoint union of sets $K_{0}, L_{0}$, and $A_{0}$, and for which $F \cap(S \cup E)=\varnothing$. Then we can construct a suitably partitioned subset $\left(S^{\prime}, e^{\prime}, K^{\prime}, L^{\prime}, A^{\prime}\right) \geqslant(S, e, K, L, A)$ such that $S \cup F \subset S^{\prime}, K \cup K_{0}=K^{\prime}$, $L \cup L_{0}=L^{\prime}, A \cup A_{0} \subset A^{\prime}, S^{\prime} \sim S$ is finite, and $S^{\prime} \sim S \subset F \cup E$.

Proof. By induction on the size of $F$, we may assume it consists of a single element $x$. If $x \in A_{0}$, set $e^{\prime}(x)=1, A^{\prime}=A \cup A_{0}, K^{\prime}=K, L^{\prime}=L$, and $S^{\prime}=S \cup F$. If $x \in K_{0}$, let $m$ be the least positive integer such that $p^{m} x=0$ or $p^{m} x \notin E$. If $p^{m} x \in S$, put $x, p x, \ldots, p^{m-1} x$ in $S^{\prime}$ and define $e^{\prime}\left(p^{i} x\right)=$ $e\left(p^{m} x\right)+m-i$ for $0<i<m$. If $p^{m} x \notin S$, put $p^{m} x$ in $A^{\prime}$, put $p^{i} x$ in $S^{\prime}$ for $0<i<m$, and define $e^{\prime}\left(p^{i} x\right)=1+m-i$. Define $S^{\prime}$ (and $\left.A^{\prime}\right)$ to include any elements we have added, in addition to $S$ (to $A$ ). Define $K^{\prime}=K \cup K_{0}$ and $L^{\prime}=L \cup L_{0}$. If $x \in L_{0}$, let $m$ be the least positive integer such that $\theta^{-1}\left(p^{m} \theta x\right)=0$ or $\theta^{-1}\left(p^{m} \theta x\right) \notin E$. Proceed as for $K_{0}$, considering $\theta^{-1}\left(p^{i} \theta x\right)$ instead of $p^{i} x$ for $0<i \leqslant m$.

Proposition 6. Suppose $(S, e, K, L, A)$ is a suitably partitioned subset of $X$ with these additional properties:

(1) if $x \in L \cup A$ and $p x \neq 0$, then there is an element $u$ in $K \cup E$ such that $p x=p u$ and $h u>h x$, and

(2) if $x \in K \cup A$ and $p \theta x \neq 0$, then there is an element $v$ in $L \cup E$ such that $p \theta x=p \theta v$ and $h \theta v>h \theta x$.

If $z \notin S$, we can construct a suitably partitioned subset ( $\left.S^{\prime \prime}, e^{\prime \prime}, K^{\prime \prime}, L^{\prime \prime}, A^{\prime \prime}\right)$ $\geqslant(S, e, K, L, A)$ which contains $z$ and for which (1) and (2) hold (with $K^{\prime \prime}, L^{\prime \prime}, A^{\prime \prime}$ replacing $\left.K, L, A\right)$.

Proof. We shall construct increasing sequences $\left\{K_{i}\right\}_{i=0}^{\infty},\left\{L_{i}\right\}_{i=0}^{\infty},\left\{A_{i}\right\}_{i=0}^{\infty}$ of subsets of $X$, such that for each $i, F_{i}=K_{i} \cup L_{i} \cup A_{i}$ is a disjoint union and $F_{i} \cap(S \cup E)=\varnothing$. In addition: 
(1') when $i$ is odd and $x \in L_{i} \cup A_{i}$ with $p x \neq 0$, there shall be an element $u$ in $K_{i+1} \cup K \cup E$ so that $p u=p x$ and $h u>h x$;

(2') when $i$ is even and $x \in K_{i} \cup A_{i}$ with $p \theta x \neq 0$, there shall be an element $v$ in $L_{i+1} \cup L \cup E$ so that $p \theta v=p \theta x$ and $h \theta v>h \theta x$.

Finally, for each $i \geqslant 0$ we shall have a suitably partitioned subset

$$
\begin{aligned}
& \left(S_{i}, e_{i}, K \cup K_{i}, L \cup L_{i}, A \cup A_{i}\right) \\
& \quad \preccurlyeq\left(S_{i+1}, e_{i+1}, K \cup K_{i+1}, L \cup L_{i+1}, A \cup A_{i+1}\right)
\end{aligned}
$$

Since $S^{*}$ satisfies (1) and (2) if $S$ does, we shall assume $S=S^{*}$, and thus by induction on the exponent of $z$, we may assume $z \notin E$. Thus both $h p z$ and $h(p \theta z)$ are limit ordinals or $\infty$.

To begin, set $F_{0}=\{z\}$ and apply Lemma 2, with $e_{0}=e, A_{0}=\{z\}$, and $K_{0}=L_{0}=\varnothing$. Then define $S_{1}=S^{\prime}, A_{1}=A^{\prime} \sim A, K_{1}=K_{0}, L_{1}=L_{0}$, and $e_{1}$ to be the extension of $e_{0}$. Given $\left(S_{i}, e_{i}, K_{i}, L_{i}, A_{i}\right)$, to define $F_{i+1}$ we proceed as follows. If $i$ is odd, consider $x$ in $L_{i} \cup A_{i}$ with $p x \neq 0$; note then $h(p x)$ is a limit ordinal. We can find an element $u$ for which $p x=p u$ and $h u>h x$, and since $F_{i}$ is finite we may assume $u \notin F_{i}$. If $u \in S \sim(K \cup E)$ then $u \in L \cup$ $A$ and so by (1), there is $u^{\prime}$ in $K \cup E$ with $p u^{\prime}=p u$ and $h u^{\prime}>h u$; thus (1') holds. If $u \notin S \cup E$, put $u$ in $K_{i+1}$ so that (1') will hold in any case. Define $K_{i+1}$ to include all elements $u$ added in this way, along with $K_{i}$. Define $L_{i+1}=L_{i}$. To define $A_{i+1}$, apply Lemma 2 to $F_{i} \cup K_{i+1}$; this gives a set $A^{\prime}$ for which $A^{\prime} \sim A$ is finite; set $A_{i+1}=A_{i} \cup\left(A^{\prime} \sim A\right)$. Now that $K_{i+1}, L_{i+1}$, and $A_{i+1}$ are defined, set $F_{i+1}$ to be their union. Apply Lemma 2 to obtain $S_{i+1}$ and $e_{i+1}$.

If $i$ is even, consider $K_{i} \cup A_{i}$ and proceed in a similar manner so that (2') will hold. It is clear that with $S^{\prime \prime}=\cup S_{i}, K^{\prime \prime}=\cup K_{i}, L^{\prime \prime}=\cup L_{i}, \mathrm{~A}^{\prime \prime}=$ $\cup A_{i}$ and $e^{\prime \prime}$ defined in the obvious way, the result follows.

A Zorn's Lemma argument yields the next result.

Proposition 7. There are subsets $K, L, A$ of $X$ and a function $e$ such that $(X, e, K, L, A)$ is suitably partitioned. In addition,

(1) if $x \in L \cup A$ with $p x \neq 0$, then there is an element $u$ in $K \cup E$ such that $p x=p u$ and $h u>h x$, and

(2) if $x \in K \cup A$ with $p \theta x \neq 0$, then there is an element $v$ in $L \cup E$ such that $p \theta x=p \theta v$ and $h \theta v>h \theta x$.

Proposition 8. The T-function $\theta$ is the composition of two strip-grafts.

Proof. Let $e, K, L, A$ be such that $(X, e, K, L, A)$ is suitably partitioned and (1) and (2) of Proposition 7 hold. For each $x$ in $X$, let $x^{\prime}=x$ and set $X^{\prime}=\left\{x^{\prime}: x \in X\right\}$, with multiplication by $p$ on $X^{\prime}$ defined by $p\left(x^{\prime}\right)=(p x)^{\prime}$ if $x \in K \cup E, p\left(x^{\prime}\right)=0$ if $x \in L \cup A$. With $\sigma_{1}: X \rightarrow X^{\prime}$ the identity function, 
Lemma 1 shows that $X^{\prime}$ is a $p$-basic tree. In view of (1), the set $\left\{x \in p^{-1} z\right.$ : $\left.p \sigma_{1}(x)=\sigma_{1}(p x)\right\}$ is dense for a nonzero $z$ in $X$. Thus, Lemma 1 shows that $\sigma_{1}$ is a stripping function.

For each $y$ in $Y$, let $y^{\prime}=y$ and set $Y^{\prime}=\left\{y^{\prime}: y \in Y\right\}$, with multiplication by $p$ on $Y^{\prime}$ defined by $p\left(y^{\prime}\right)=(p y)^{\prime}$ if $\theta^{-1} y \in L \cup E, p\left(y^{\prime}\right)=0$ if $\theta^{-1} y \in K$ $\cup A$. Then $Y^{\prime}$ is a $p$-basic tree and the identity function $\sigma_{2}: Y \rightarrow Y^{\prime}$ is a stripping function.

Now define $Z=\{(x, y) \in X \times Y: y=\theta x\}$, with multiplication by $p$ in $Z$ defined as $p(x, y)=(p x, \theta(p x))$ if $x \in K \cup E ; p(x, y)=\left(\theta^{-1} p y, p y\right)$ if $x \in L \cup E$, and $p(x, y)=(0,0)$ if $x \in A$. An easy induction shows that $e(x)$ is the exponent of the element $(x, y)$ in $Z$, so that $Z$ is a $p$-basic tree. If $\alpha$ is the height of $(x, y)$ in $Z$, an induction on $\alpha$ shows that $\alpha$ is the common value $h x=h y$.

Let $\sigma_{3}: Z \rightarrow X^{\prime}$ be defined by $\sigma_{3}(x, y)=x^{\prime}$, and $\sigma_{4}: Z \rightarrow Y^{\prime}$ be defined by $\sigma_{4}(x, y)=y^{\prime}$. Note $p \sigma_{3}(x, y)=\sigma_{3}(p(x, y))$ for $x$ in $K \cup E$, whereas $p \sigma_{3}(x, y)$ $=0$ for $x$ in $L \cup A$. In view of this and (1), the set $\left\{(u, v) \in p^{-1}(x, y)\right.$ : $\left.p \sigma_{3}(u, v)=\sigma_{3}(p(u, v))\right\}$ is dense in $p^{-1}(x, y)$ for nonzero $(x, y)$. Consequently, $\sigma_{3}$ is a stripping function; similarly, $\sigma_{4}$ is a stripping function. Setting $\xi_{2}=\sigma_{3}^{-1} \sigma_{1}$ and $\xi_{1}=\sigma_{2}^{-1} \sigma_{4}$, we observe that $\theta=\xi_{1} \xi_{2}$, which completes the proof.

We now drop the assumption that $X$ and $Y$ are fully stripped. The remaining results follow easily from the preceding discussion.

THeOREM 4. The $p$-basic trees $X$ and $Y$ are equivalent iff there is a p-basic tree $Z$ such that:

(1) the natural direct sum decompositions of $[X]$ and $[Z]$ have isomorphic refinements, and

(2) the natural direct sum decompositions of $[Y]$ and $[Z]$ have isomorphic refinements.

TheOREM 5 (Ulm's THEOREM FOR P-BASIC TREES). Two $p$-basic trees are equivalent if and only if they have the same Ulm invariants.

COROllary (Ulm's THEOREM FOR SIMPLY PRESENTED GROUPS). Two simply presented groups are isomorphic if and only if they have the same Ulm invariants.

\section{REFERENCES}

1. P. Crawley and A. W. Hales, The structure of abelian p-groups given by certain presentations, J. Algebra 12 (1969). 10-23. MR 39 \#307.

2. The structure of abelian p-groups given by certain presentations. II, J. Algebra 18 (1971), 264-268. MR 43 \#2076.

3. L. Fuchs, Infinite abelian groups, Academic Press, New York, 1970. MR 41 \#333.

4. P. Hill, On the classification of Abelian groups, (to appear). 
5. Ulm's theorem for totally projective groups, Notices Amer. Math. Soc. 14 (1967), 940. Abstract \#652-15.

6. G. Kolettis, Jr., Direct sums of countable groups, Duke Math. J. 27 (1960); 111-125. MR 22 \# 1616.

7. H. Ulm, Zur Theorie der Abzählbar-unendlichen Abelschen Gruppen, Math. Ann. 107 (1933), $774-803$.

8. E. A. Walker, The groups $P_{\beta}$, Symposia Mathematica, Vol. 13 (Convegno di Gruppi Abeliani, INDAM, Rome, 1972), Academic Press, London, 1974, 245-255. MR 51 \# 751.

Department of Mathematics, University of Colorado, Colorado Springs, Colorado 80907 